\title{
Road Maintenance Priority Based On Multi-Criteria Approach (Case Study of Bali Province, Indonesia)
}

\author{
Putu Alit Suthanaya ${ }^{1}$ \\ ${ }^{1}$ Department of Civil Engineering, Faculty of Engineering, Udayana University, Bali, Indonesia \\ suthanaya@rocketmail.com
}

\begin{abstract}
Total length of provincial roads in the Province of Bali is $860.53 \mathrm{~km}$ spread over nine regions. As very limited budget available for road maintenance projects, it is required to prioritize the road segments to be maintained. Within the framework of sustainable development, socio-cultural aspect is also important to be considered. The objectives of this study are to determine the hierarchy and quantitative measures for each of the criteria in determining the scale of priority in maintaining the provincial roads by incorporating socio-cultural aspect. By applying Analytical Hierarchy Process (AHP), it was found that the stakeholders for road development in Bali Province gave priority to road condition 5.36 times more than socio-culture accessibility. They also gave priority to traffic condition $\mathbf{2}$ times more than socio-culture accessibility. The priority of socio-culture accessibility is 1.38 times more than institutional aspect. The stakeholders in Bali Province consider the road condition as the main factor for determining road development priority. While the socio-culture accessibility only accounted for $11 \%$ of the total score. Based on the percentile method, it was identified that 28 road segments were in the very high priority category, 20 road segments were in the high priority category, 36 road segments were in the medium priority category and the rest were in the low priority category.
\end{abstract}

Keyword-AHP, Multi-criteria, Priority, Road maintenance

\section{INTRODUCTION}

Road network is an important transport infrastructure to support economic and social development in all countries. A good road network and pavement performance are essential for stable traffic movement. Almost all countries, in particular developing countries face very limited budget available for road maintenance. This condition is also faced by the government at the provincial level such as in Bali Province, Indonesia. The government of Bali Province has experienced difficulties in distributing limited funds available for road maintenance. The existing method adopted by the government of Bali Province to rank the road segment maintenance is mainly based on the road pavement condition only. Difficulties were faced to prioritize many road segments with the same damage condition. Public pressure has increased from year to year on the problem of prioritization of road maintenance. Therefore, reliable and transparent methods are required in order to rank the road maintenance projects.

A major and widely adopted methodological framework for road project appraisal in developed and developing countries are the Cost-Benefit Analysis (CBA) [1]. The CBA expresses known or estimated costs and benefits of a project in monetary terms, discounts them to a reference point in time, and compares to check whether benefit outweighs costs. Several researchers have criticized the application of CBA in project evaluation. CBA does not incorporate the stakeholder's opinion and the analysis is focused only on the monetary value [2]. It becomes more and more problematic in considering a wide range of decision-making criteria. The CBA has some ethical limitations that may be overcome by using a Multi-Criteria Analysis (MCA).

The MCA has been widely used as it can incorporate various aspects. Other methods, such as EconomicEffects Analysis (EEA), private investment analysis and CBA, only consider economic aspect without incorporating other aspects such as environmental and social aspects [3]. The road projects should be chosen within the concept of sustainable development by integrating economic development, social development and environmental protection [1]. In order to pursue this principle, criteria and measures need to be developed to contrast and rank road maintenance projects.

The multi-criteria analysis provides the possibility to incorporate decision maker's point of view in the evaluation of transport project. The difficulties in the application of multi-criteria analysis are in determining the criteria weights. Different decision maker tends to have a different opinion and different weight on a certain criterion. One of the multi-criteria methods that are widely adopted in the transport project is Analytical Hierarchy Process (AHP) [4]. AHP considers both qualitative and quantitative multi-criteria in the decisionmaking process [5]. This study applied AHP in order to prioritize provincial road maintenance projects. The objectives of this research are to develop criteria and weighting these criteria by incorporating socio-cultural aspect in determining provincial road maintenance priority in Bali Province, Indonesia. 


\section{MULTI-CRITERIA APPROACH}

\section{A. Previous Studies}

Several methods are available for the evaluation of the transport projects, for example, Analytic Hierarchy Process (AHP), Analytic Network Process (ANP), REGIME, ELECTRE, Multi-Attribute Utility, etc. [6]. Other researcher stated that numerous methods available that can be applied for conducting multi-criteria analysis, such as: SAW (Simple Additive Weighting Method), TOPSIS (The Technique for Order of Preference by Similarity to Ideal Solution), ELECTRA (Elimination Et Choix Traduinsant Realite), PROMETHEE (A Preference Ranking Organization Method for Enrichment Evaluation), VIKOR, etc. [5].

A study in Lithuania applied multi-objective optimization based on ratio analysis method (MOORA method) in order to optimize road design alternatives [7]. Highways rating system has been developed based on discrete multi-criteria analysis [8]. They combined technical and economic factors to decide highway priority development. Variables considered include, present day traffic demand in terms of PCU, the average percentage composition of heavy traffic, vehicle damage factor, average journey speed in the desired stretch, growth in per capita income, net district domestic product, accident cost and road users cost. A composite index has been developed by applying Analytical Hierarchy Process (AHP) to rank 20 District Roads in Kerala, India [9]. They developed a Multi-Criterion Decision Model by incorporating several aspects, such as Growth centers, Road utilization, Connectivity, Accessibility, Backwardness and the number of Commercial vehicles. A study in Europe has applied multi-criteria analysis in evaluating road designs [5]. Another study has also used AHP in solving problems in the area of transport [10].

\section{B. Analytic Hierarchy Process (AHP)}

The Analytic Hierarchy Process (AHP) has been claimed as an effective method to solve a complex decision-making and can be used by the decision maker to prioritize projects [4]. The AHP can help decision maker to identify subjective and objective criteria. The consistency of the decision maker's point of view can be evaluated in order to avoid bias. Evaluation criteria can be developed and the best decision can be made. The weight for each criterion can be calculated based on the decision maker's opinion on that criterion. A high value of weight represents the importance of the criterion. The scores for each alternative can be calculated. A high score represents a good performance of the alternative for a certain criterion. The last steps, the criteria weights, and the alternative scores are combined in order to determine the total score and the ranking for each option. The total score for an alternative is a weighted sum of the scores by considering all criteria [4]. The process of the AHP evaluation includes calculation of the vector of criteria weights, calculation of the matrix of alternative scores and ranking the alternatives as described as follow [4]:

1) Calculation of the Vector of Criteria Weights: Calculation of the weights for each criterion is started by developing a pairwise comparison matrix $\mathbf{A}$. The matrix $\mathbf{A}$ is a $m \times m$ matrix ( $m$ is the number of criteria). The entry $a_{j k}$ of the matrix indicates the importance of the $j$ th criterion compared to the $k$ th criterion. If $a_{j k}>1$, then the $j$ th criterion is said to be more important than the $k$ th criterion, while if $a_{j k}<1$, then the $j$ th criterion is less important than the $k$ th criterion. If the importance of the two criteria is the same, then the entry $a_{j k}$ is 1 . The value of $a_{j k}$ and $a_{k j}$ is as follow [4]:

$$
a_{j k} \cdot a_{k j}=1
$$

Where, $a_{j j}=1$ for all $j$. The importance between two criteria is identified based on a numerical scale from 1 to 9. After the matrix $\mathbf{A}$ is built, the normalized pairwise comparison matrix $\mathbf{A}_{\text {norm }}$ is obtained by making equal to 1 the sum of the entries on each column, i.e. each entry $\bar{a}_{j k}$ of the matrix $\mathbf{A}_{n o r m}$ is calculated as [4]:

$$
\bar{a}_{j k}=\frac{a_{j k}}{\sum_{l=1}^{m} a_{l k}}
$$

Later, the criteria weight vector $\mathbf{w}$ is calculated by averaging the entries on each row of $\mathbf{A}_{\text {norm }}$, as follow [4]:

$$
w_{j}=\frac{\sum_{l=1}^{m} \bar{a}_{j l}}{m}
$$

2) Calculating the Matrix of Alternative Scores: The matrix of alternative scores is a $n \times m$ matrix $\mathbf{S}$. The entry $s i j$ of $\mathbf{S}$ indicates the score of the $i$ th alternative with respect to the $j$ th criterion. The scores is obtained based on a pairwise comparison matrix which is developed for each of the $m$ criteria, $j=1, \ldots, m$. The matrix is a $n \times n$ matrix, where $n$ is the number of alternatives evaluated. Every entry of the matrix indicates the evaluation of the $i$ th alternative relative to the $h$ th alternative by considering the $j$ th criterion. If two alternatives are evaluated as equivalent considering the $j$ th criterion, then the entry is 1 . The constraint for entries $b_{i h}^{(j)}$ and $b_{h i}^{(j)}$ is as follow [4]: 


$$
b_{i h}^{(j)} \cdot b_{h i}^{(j)}=1
$$

and $b_{i i}^{(j)}=1$ for all $i$.

Next, the AHP applies to each matrix $\mathrm{B}^{(j)}$ following similar procedure for the pairwise comparison matrix $\mathbf{A}$, i.e. it divides each entry by the sum of the entries, and then it averages the entries, hence obtaining the score vectors $\mathbf{S}^{(j)}, j=1, \ldots, m$. The vector $\mathbf{S}^{(j)}$ contains the evaluated alternatives with respect to the $j$ th criterion. The score matrix $\mathbf{S}$ is calculated as [4]:

$$
\mathbf{S}=\left[\mathbf{s}^{(1} \ldots \mathbf{S}^{(m)}\right]
$$

i.e. the $j$ th column of $\mathbf{S}$ corresponds to $\mathbf{S}^{(j)}$.

3) Ranking the Alternatives: After the weight vector $\boldsymbol{w}$ and the score matrix $\mathbf{S}$ have been calculated, a vector $\mathbf{v}$ of total scores is calculated by multiplying $\mathbf{S}$ and $\mathbf{w}$, i.e. [4]:

$$
\boldsymbol{v}=\mathbf{S} \cdot \boldsymbol{w}
$$

The $i$ th entry $v i$ of $\mathbf{v}$ indicates the total score for the $i$ th alternative. Finally, the rank of alternatives is determined by ordering the total scores in decreasing order.

4) Evaluating the Consistency: The Consistency Index $(C I)$ is evaluated by calculating the scalar $x$ as the average of the elements of the vector, where $j$ th element is the ratio of the $j$ th element of the vector $\mathbf{A} \cdot \boldsymbol{w}$ to the corresponding element of the vector $w$ [4], where,

$$
C I=\frac{x-m}{m-1}
$$

The value of $C I=0$ indicates a perfect consistent decision maker but small inconsistency may be tolerated with the value of $\mathrm{CI} / \mathrm{RI}<0.1$ (RI is the Random Index, i.e. the consistency index when the entries of $\mathrm{A}$ are completely random).

\section{Methodology}

Fig. 1 shows the road network in Bali Province. The total length of road in Bali Province reached 7,843.98 $\mathrm{km}$, consists of $535.23 \mathrm{~km}$ national roads, $860.53 \mathrm{~km}$ provincial roads and $6,448.22 \mathrm{~km}$ district roads [11]. From $860.53 \mathrm{~km}$ provincial roads, $52.16 \%$ is in a good condition, $22.22 \%$ is in medium condition and $25.62 \%$ is in poor condition. The provincial roads were divided into 150 road segments.

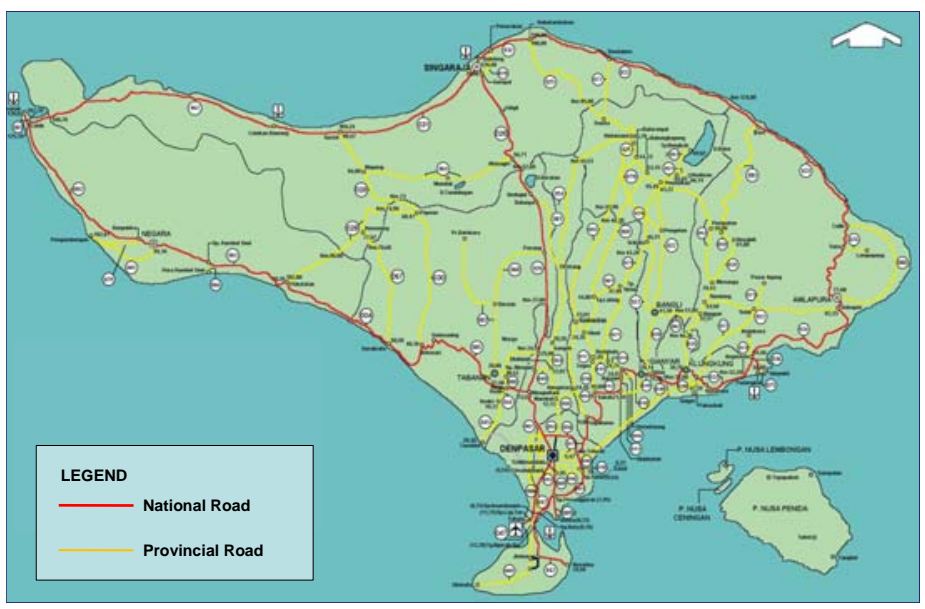

Fig. 1. Road network in Bali Province

In order to determine the priority of the road segment to be maintained based on the AHP method, there was three stages process: first, constructing the hierarchy; second, weighting the indicators based on a pair-wise comparison, and third, determining the final value for the alternatives. A hierarchical structure was developed with the goal in the first level, the primary criterion in the second level, and the sub-criterion in the third level.

Hierarchy level-1 is the goal, i.e. to determine the priority of road segments to be maintained. For hierarchical level-2 is grouped into four groups of criteria that affect the goal, i.e. institutional aspect (A), road network aspect (B), socio-culture accessibility aspect (C), and traffic aspect (D). At level 3, each of the subsystem in level 2 was divided further as the consequences of criterion division on the second level. The criterion group Institutional Aspect was divided into the inclusion of the road into the strategic planning (a1) and proposed by the community (a2). The criterion group Road Condition was divided into pavement condition (b1) and road function (b2). The criterion group Socio-culture accessibility was divided into accessibility to tourism location (c1), accessibility to office area (c2), accessibility to mining area (c3) and accessibility to the temple (holy places) (c4). The criterion group traffic condition was influenced by traffic volume (ADT) (d). 
After developing the hierarchy and criteria, a questionnaire was developed to help experts in ranking each criterion based on its relative importance using a nine-point Likert scale. The respondents were selected based on the purposive sampling method. This method was used with consideration because not all elements / members of an institution or community members understand and engage directly in the decision-making related to the preparation of priority for road segment maintenance in the Bali Province. Respondents selected in this study consisted of the experts in the field of transportation including practitioners, academics, legislative membership, professional organizations and civil society organizations.

\section{RESULTS AND DISCUSSION}

\section{A. Pairwise Comparison on the Second Level}

Determination of the weights for each criterion was started by developing a pairwise comparison matrix. Table 1 shows a pairwise comparison matrix for the second level. At this level, there were four criteria considered, i.e. institutional aspect (A), a road condition (B), socio-culture accessibility (C), traffic (D). The relative importance of two criteria was measured based on a numerical scale from 1 to 9 . A reciprocal matrix was developed from the pairwise comparison. After developing a comparison matrix, the next step was to determine priority vector, which is normalized Eigen vector of the matrix. Eigen vector was calculated by normalizing each column of the matrix as follow: $\rho \mathrm{A}=1.00 \times 0.19 \times 0.50 \times 0.30=0.03$, the results for $\rho \mathrm{B}$, $\rho \mathrm{C}$ and $\rho \mathrm{D}$ are presented in Table 1 .

The normalized principal Eigen vector or priority vector was calculated by averaging across the rows. The priority vector shows the weights among the criteria compared. The criteria weight vector W was built by averaging the entries on each row. For example, WA $=4 \sqrt{ } 0.03=0.42$. The results of $\mathrm{WB}, \mathrm{WC}$ and $\mathrm{WD}$ can be seen in Table 1. Finally, the weight of each criterion (Xi) is calculated. For example XA $=0.42 / 5.49=0.08$. The results of $\mathrm{XB}, \mathrm{XC}$ and $\mathrm{XD}$ can be seen in Table 1. It can be seen that the highest weight is obtained for criterion $\mathrm{B}$ (road condition) with XB value of 0.59 , followed by criterion $\mathrm{D}$ (traffic condition), criterion $\mathrm{C}$ (socio-culture accessibility) and criterion $\mathrm{A}$ (institutional aspect).

Base on the results presented in Table 1, the stakeholders for road development in Bali Province gave priority to road condition $59 / 11=5.36$ times more than socio-culture accessibility. They also gave priority to traffic condition $22 / 11=2$ times more than socio-culture accessibility. The priority of socio-culture accessibility is 1.38 times more than institutional aspect. It can be concluded that the stakeholders in Bali Province consider the road condition as the main factor for determining provincial road maintenance priority. While the socio culture accessibility only accounted for $11 \%$ of the total score.

TABLE I. Pairwise Comparison Matrix on the Second Level

\begin{tabular}{|l|l|l|l|l|l|l|l|}
\hline Criteria & A & B & C & D & $\rho \mathrm{i}$ & Wi & Xi \\
\hline A & 1.00 & 0.19 & 0.50 & 0.30 & 0.03 & 0.42 & 0.08 \\
\hline B & 5.26 & 1.00 & 4.80 & 4.40 & 111.16 & 3.25 & 0.59 \\
\hline C & 2.00 & 0.21 & 1.00 & 0.36 & 0.15 & 0.62 & 0.11 \\
\hline D & 3.33 & 0.23 & 2.78 & 1.00 & 2.10 & 1.20 & 0.22 \\
\hline$\sum$ & & & & & & 5.49 & 1.00 \\
\hline
\end{tabular}

In order to evaluate the consistency of the Eigen vector, the Eigen maximum $(\lambda$ max $)$ was calculated first. For example, $\lambda \max \mathrm{A}=(1.00 \times 0.08)+(0.19 \times 0.59)+(0.50 \times 0.11)+(0.30 \times 0.22)=0.31$. Later, $\lambda \max \mathrm{B}, \mathrm{C}$ and $\mathrm{D}$ were calculated in the same way and the results as shown in Table 2 . The value of the $\lambda$ max is 4.2 , therefore, $\mathrm{IC}=(4.2-4) /(4-1)=0.073$. For number of criterion $4, \mathrm{RI}=0.9$, therefore, $\mathrm{CR}=0.073 / 0.9=0.081<$ $10 \%$, which indicates a consistent result.

TABLE II. Calculation of Eigen Maximum $(\lambda \max )$

\begin{tabular}{|c|c|c|c|c|c|c|c|c|}
\hline Criteria & $\mathrm{A}$ & $\mathrm{B}$ & $\mathrm{C}$ & $\mathrm{D}$ & \multirow{6}{*}{$\mathrm{X}$} & $\mathrm{Xi}$ & \multirow{6}{*}{$=$} & $\lambda \max$ \\
\hline A & 1.00 & 0.19 & 0.50 & 0.30 & & 0.08 & & 0.31 \\
\hline B & 5.26 & 1.00 & 4.80 & 4.40 & & 0.59 & & 2.50 \\
\hline $\mathrm{C}$ & 2.00 & 0.21 & 1.00 & 0.36 & & 0.11 & & 0.47 \\
\hline $\mathrm{D}$ & 3.33 & 0.23 & 2.78 & 1.00 & & 0.22 & & 0.92 \\
\hline$\sum$ & & & & & & 1.00 & & 4.20 \\
\hline
\end{tabular}

\section{B. Pairwise Comparison on the Third Level (3a)}

Table 3 shows a pairwise comparison matrix for the third level (3a). At this level, there were two criteria considered, i.e. The Inclusion in Strategic Planning (a1) and Proposed by Community (a2). It can be seen that the highest weight is obtained for criterion a1 with X(a1) value of 0.69 , whilst only 0.31 for X(a2). Based on the 
results presented in Table 3, the stakeholders for road maintenance in Bali Province gave priority to the inclusion of road in the strategic planning 69/31=2.23 times more than if the road proposed by the community.

TABLE III. Pairwise Comparison on the Third Level (3a)

\begin{tabular}{|l|l|l|l|l|l|}
\hline Criteria & a1 & a2 & pi & Wi & Xi \\
\hline a1 & 1.00 & 2.20 & 2.20 & 1.48 & 0.69 \\
\hline a2 & 0.45 & 1.00 & 0.45 & 0.67 & 0.31 \\
\hline$\sum$ & & & & 2.15 & 1.00 \\
\hline
\end{tabular}

\section{Pairwise Comparison on the Third Level (3b)}

Table 4 shows a pairwise comparison matrix for the third level (3b). At this level, there were two criteria considered, i.e. Road condition (b1) and Road function (b2). It can be seen that the highest weight is obtained for criterion b1 (Road condition) with $\mathrm{X}(\mathrm{b} 1)$ value of 0.77 , whilst only 0.23 for $\mathrm{X}(\mathrm{b} 2)$. Based on the results presented in Table 3, the stakeholders for provincial road maintenance in Bali Province gave priority to Road condition $77 / 23=3.35$ times more than Road function.

TABLE IV. Pairwise Comparison on the Third Level (3b)

\begin{tabular}{|l|l|l|l|l|l|}
\hline Criteria & b1 & b2 & pi & Wi & Xi \\
\hline b1 & 1.00 & 3.40 & 3.40 & 1.85 & 0.77 \\
\hline b2 & 0.29 & 1.00 & 0.29 & 0.54 & 0.23 \\
\hline$\sum$ & & & & 2.39 & 1.00 \\
\hline
\end{tabular}

\section{Pairwise Comparison on the Third Level (3c)}

Table 5 shows a pairwise comparison matrix for the third level (3c). At this level, there were four criteria considered, i.e. accessibility to tourism location (c1), accessibility to office area (c2), accessibility to mining location (c3), and accessibility to temple location (c4). It can be seen that the highest weight is obtained for criterion $\mathrm{c} 1$ (accessibility to tourism location) with $\mathrm{Xc1}$ value of 0.54 , followed by criterion 4 (accessibility to temple location), criterion c2 (accessibility to office area) and criterion c3 (accessibility to mining location). Based on the results presented in Table 5, the stakeholders for provincial road maintenance in Bali Province gave priority to accessibility to tourism location $54 / 20=2.7$ times more than accessibility to temple location. Based on this results, it can be concluded that the stakeholders in Bali Province consider accessibility to tourism location as the main factor for determining road maintenance priority at this level. While accessibility to temple location is only accounted for $20 \%$ of the total score.

Table 6 shows the calculation of the Eigen maximum $(\lambda \max )$. The value of the $\lambda$ max is 4.26 , therefore, IC $=$ $(4.26-4) /(4-1)=0.086$. For number of criterion $4, \mathrm{RI}=0.9$, therefore, $\mathrm{CR}=0.086 / 0.9=0.096<10 \%$, which indicates a consistent result.

TABLE V. Pairwise Comparison on the Third Level (3c)

\begin{tabular}{|l|l|l|l|l|l|l|l|}
\hline Criteria & $\mathrm{c} 1$ & $\mathrm{c} 2$ & $\mathrm{c} 3$ & $\mathrm{c} 4$ & $\rho \mathrm{i}$ & $\mathrm{Wi}$ & $\mathrm{Xi}$ \\
\hline $\mathrm{c} 1$ & 1.00 & 4.00 & 5.00 & 2.60 & 52.00 & 2.69 & 0.54 \\
\hline $\mathrm{c} 2$ & 0.25 & 1.00 & 4.40 & 0.45 & 0.50 & 0.84 & 0.17 \\
\hline $\mathrm{c} 3$ & 0.20 & 0.23 & 1.00 & 0.88 & 0.04 & 0.45 & 0.09 \\
\hline $\mathrm{c} 4$ & 0.35 & 2.22 & 1.14 & 1.00 & 0.88 & 0.99 & 0.20 \\
\hline$\sum$ & & & & & & 4.97 & 1.00 \\
\hline
\end{tabular}

TABLE VI. Calculation of Eigen Maximum $(\lambda \max )$

\begin{tabular}{|c|c|c|c|c|c|c|c|c|}
\hline Criteria & $\mathrm{c} 1$ & $\mathrm{c} 2$ & c3 & $\mathrm{c} 4$ & \multirow{6}{*}{$X$} & $\mathrm{Xi}$ & \multirow{6}{*}{$=$} & $\lambda \max$ \\
\hline $\mathrm{c} 1$ & 1.00 & 4.00 & 5.00 & 2.60 & & 0.54 & & 2.19 \\
\hline $\mathrm{c} 2$ & 0.25 & 1.00 & 4.40 & 0.45 & & 0.17 & & 0.79 \\
\hline c3 & 0.20 & 0.23 & 1.00 & 0.88 & & 0.09 & & 0.41 \\
\hline $\mathrm{c} 4$ & 0.35 & 2.22 & 1.14 & 1.00 & & 0.20 & & 0.87 \\
\hline$\sum$ & & & & & & 1.00 & & 4.26 \\
\hline
\end{tabular}

\section{E. Recapitulation of the Quantitative Value for Each Criterion}

Table 7 shows recapitulation of quantitative value for each criterion with score interval $0-1000$. It can be seen that the main criteria in determining road development priority are road condition, followed by traffic condition, road function, accessibility to tourism location, inclusion in the strategic planning, proposed by the 
community, accessibility to temple location, accessibility to the office area and accessibility to the mining area. The weight of each criterion was then applied to rank the road segments. Based on the percentile method, it was found that 28 road segments were in the very high priority category, 20 road segments were in the high priority category, 36 road segments were in the medium priority category and the rest were in the low priority category.

TABLE VII. Recapitulation of the Quantitative Value for Each Criterion

\begin{tabular}{|r|l|c|c|c|}
\hline No & \multicolumn{1}{|c|}{ Criteria } & $\begin{array}{c}\text { Analysis } \\
\text { Code }\end{array}$ & Weight & $\begin{array}{c}\text { Conversion } \\
\mathbf{x} 1000\end{array}$ \\
\hline 1 & Inclusion in the Strategic Planning (Renstra) & $\mathrm{a} 1$ & $5.50 \%$ & 55 \\
\hline 2 & Proposed by community (Musrenbang) & $\mathrm{a} 2$ & $2.50 \%$ & 25 \\
\hline 3 & Road condition & $\mathrm{b} 1$ & $45.40 \%$ & 454 \\
\hline 4 & Road function & $\mathrm{b} 2$ & $13.60 \%$ & 136 \\
\hline 5 & Accessibility to tourism location & $\mathrm{c} 1$ & $5.90 \%$ & 59 \\
\hline 6 & Accessibility to office area & $\mathrm{c} 2$ & $1.90 \%$ & 19 \\
\hline 7 & Accessibility to mining area & $\mathrm{c} 3$ & $1.00 \%$ & 10 \\
\hline 8 & Accessibility to temple location & $\mathrm{c} 4$ & $2.20 \%$ & 22 \\
\hline 9 & Traffic condition & $\mathrm{d}$ & $22.00 \%$ & 220 \\
\hline & T O T A L & $100.00 \%$ & 1000 \\
\hline
\end{tabular}

\section{CONCLUSION}

Due to budget limitation available for the provincial road maintenance in Bali Province, it is required to develop a method to prioritize road maintenance projects. This study has applied Analytical Hierarchy Process (AHP) in order to accommodate multi-criteria faced by the decision makers. Three levels of the hierarchy have been constructed by incorporating socio-cultural aspect. It was found that the highest weight was obtained for criterion B (road condition) with XB value of 0.59 , followed by criterion D (traffic condition), criterion C (socio-culture accessibility) and criterion A (institutional aspect). The stakeholders for road development in Bali Province gave priority to road condition 5.36 times more than socio-culture accessibility. They also gave priority to traffic condition 2 times more than socio-culture accessibility. The priority of socio-culture accessibility is 1.38 times more than institutional aspect. The stakeholders in Bali Province consider the road condition as the main factor for determining road development priority. While the socio -culture accessibility only accounted for $11 \%$ of the total score. Based on the percentile method, it was identified that 28 road segments were in the very high priority category, 20 road segments were in the high priority category, 36 road segments were in the medium priority category and the rest were in the low priority category.

\section{REFERENCES}

[1] S. B. Bhandari, P. B. Shahi, P.B. and R. N. Shrestha, "Ranking rural road projects: Weighting different evaluation criteria with a focus on the case of Nepal," International Journal of Engineering Research and Science \& Technology, vol. 5 (1), pp. 49-69, 2016.

[2] K. De Brucker, C. Macharis, and A. Verbeke, "Multi-criteria analysis in transport project evaluation: an institutional approach," European Transport, vol. 47, pp. 3-24, 2011.

[3] J. Dodgson, M. Spackman, A. Pearman, and L. Phillips, "Multi-criteria analysis; a manual," London School of Economics and Political Sciences, Department of Economic History, 2009.

[4] T. L. Saaty, The Analytic Hierarchy Process, McGraw-Hill, New York, 1980.

[5] L. T. Kuzovic, B. Aleksic, and D.N. Glavic, "The application of the multi-criteria analysis in evaluating of the road designs," TehnikaMenadzment, vol. 65 (1), pp. 143-150, 2015.

[6] P. Beria, I. Maltese, and I, Mariotti, "Multi-criteria analysis versus cost-benefit analysis: a comparative perspective in the assessment of sustainable mobility," European Transport Research, vol. 4, pp. 137-152, 2012.

[7] W. K. M. Brauers, F. Peldschus, E. K. Zavadskas, and A. Turskis, "Multi-objective optimization of road design alternatives with an application of the moora method," $25^{\text {th }}$ International Syposium on Automation and Robotics in Construction (ISARC), June 26-29, Institute of Internet and Intelligent Technologies, Vilnius Gediminas Technical University, Lithuania. http://www.isarc2008.vgtu.lt/, 2008.

[8] M. J. B. Alam, A.A.M. Ahmed, and A. Ahmed, "A techno-economic rating system for highways using discrete multi-criteria analysis," Journal of Civil Engineering and Construction Technology, vol. 2 (1), pp. 1-5, 2011.

[9] D. Linaraj, B.I. Koshy, and J. Pradeep, "Prioritisation of road corridors by developing composite index," International Journal of Innovative Research in Science, Engineering and Technology, vol. 2 (1), 2013.

[10] D. Baric and M. Starcevic, "Implementation of analytic hierarchy process in solving transport problems," International Journal of the Analytic Hierarchy Process, vol. 7 (2), 2015.

[11] Bali Province Bureau of Statistics. Bali in Figures 2015, Bureau of Statistics, Bali, 2016.

\section{AUTHOR PROFILE}

Putu Alit Suthanaya is a Professor in Udayana University, Indonesia. He obtained his master degree in the School of Civil and Environmental Engineering, University of New South Wales, Australia in 1998 and completed Ph.D in the area of Urban Transportation at the same place in 2003. His research interests include urban transport planning, road safety and environmental impact assessment. 